\title{
THE EFFECT OF IODINE BY MOUTH ON THE REACTION TO INTRAVENOUS INJECTIONS OF THYROXIN
}

\author{
BY CYRUS C. STURGIS, SALVADOR ZUBIRAN, GUY W. WELLS AND \\ THEODORE BADGER
}

(From the Medical Clinic of the Peter Bent Brigham Hospital)

(Received for publication November 2, 1925)

Repeated observations within recent years have shown that the oral administration of iodine will usually be followed by a striking reduction in the basal metabolism in patients with exophthalmic goiter. A histologic study of the thyroid glands which have been removed from these patients gives evidence that definite involutional changes follow the use of iodine which indicate an anatomic regression toward a normal or colloid resting state (1). It has, therefore, been assumed on very good evidence that the anatomic alteration of the gland, in part, at least, explains the reduction in the basal metabolism and the associated improvement in the patient's condition. Another possible explanation of this phenomenon is that iodine may have a direct effect, in some unknown manner, on the circulating product of the thyroid gland whether the latter is an excessive or abnormal secretion or both. In order to obtain information bearing on the action of iodine on a pure excess of the secretion of the thyroid gland, the following experimental studies were carried out.

In brief, the plan of experimentation consisted in determining the effect of intravenous injections of thyroxin in rabbits as indicated by changes in the basal metabolism, pulse-rate, and body-weight. Following this the observations were repeated with similar intravenous injections of thyroxin, after the animals had been given iodine orally in the form of Lugol's solution. Under these conditions an opportunity was afforded to observe if iodine by mouth can alter, in any way, the animals' response to thyroxin.

\section{METHODS}

The animals used were healthy, male rabbits of various breeds. They were of different ages, although an attempt was made to secure about six months old rabbits; about one-half of these animals were 
of this age. During the course of the experiments, which usually extended over long periods, several animals developed "snuffles." As long as the animal's body temperature remained normal, this had no apparent effect on the body-weight, pulse rate, or basal metabolism as indicated by the control animals. If the temperature rose above normal the experiment was immediately terminated. The animals were kept in ordinary cages and fed a liberal diet consisting of oats and "greens" which was roughly measured. A record was kept of all the food given which was not consumed.

The heart rate was determined by means of an electrocardiograph tracing which was necessary as the normal rate of a rabbit varies between 200 and 250 per minute, and in some instances, following the thyroxin injections, it rose to over 400 beats per minute. In order to keep the animals quiet during the pulse-rate determination it was necessary to restrain them on an animal board, which was not ideal as the heart rate was probably accelerated by these manipulations. For comparative studies, however, this was satisfactory as indicated by the control animals in whom the heart rate remained very constant.

The basal metabolism was calculated from the oxygen consumption, which was determined by means of a closed chamber apparatus comprised of an animal chamber with a water seal, a bell of 8 liters capacity suspended in water, an electric blower, and a soda-lime container for the removal of carbon dioxide. The chamber and movable bell which recorded the contraction of air in the circuit and as much more of the apparatus as possible were enclosed in a water bath. The chamber and bell were provided with thermometers, reading in hundredths of a degree centigrade, in order to record if the temperature in the circuit was constant throughout. A small blower in the circuit was connected by a direct shaft to an electric motor which provided a ventilation of approximately 20 liters per minute. Air leaks in the system gave very little difficulty after all the connections were once made tight. Each day, however, a test of tightness was made on the apparatus before the experiment was begun. To avoid any accumulation of carbon dioxide in the system the soda-lime was changed at suitable intervals, and as a further check the contained air, following an experment, was forced through a solution of barium 
hydroxide in order to detect the slightest trace of carbon dioxide. In no instance was there any evidence that the $\mathrm{CO}_{2}$ was not being removed efficiently. The accuracy of the apparatus was determined by frequent alcohol check tests (2) and a variation averaging 5 per cent was found. As this error was constant and indicated that the observed oxygen consumption was always 5 per cent less than the theoretical, it was considered satisfactory for our purposes. As the animals were confined in a chamber in which it was impossible to see them move, it was necessary to use a suitable apparatus to record muscular movements. This was accomplished, in all but the first few experiments, by means of a recording pen which was attached to the movable spirometer bell. Each time the animals moved a graphic record was made on a revolving drum and thereby a permanent record of muscular movements was obtained. While the activity of different animals varied, it may be said that in general they were quiet, and it was usually possible to obtain satisfactory periods. In each instance in which the animals moved a significant amount, it has been noted and considered in the interpretation of the results. The animals were fed each day at about 5 p.m., and if any portion of the food remained in the cage the following morning its presence was noted and it was. removed several hours before the metabolism determination. The procedure followed in determining the metabolism was to place the animal in the chamber for a preliminary period of one-half to one hour. After conditions were constant, and the animal quiet, the oxygen consumption was recorded for two fifteen minute periods. In almost all instances these periods agreed within 5 per cent. After having obtained the oxygen consumption, the figures were changed to calories, by the assumption of a respiratory quotient of 0.92 , and by reference to the table of Zuntz and Schumburg (3) which gives the calorific value of a liter of oxygen for various non-protein respiratory quotients. The results were then expressed in per cent of normal by use of the average normal values of heat production per kilo in rabbits given by $\mathrm{E}$. Voit (4). Although some question may arise concerning correctness of the assumed respiratory quotients and normal values of heat production for rabbits which were used, this has no bearing on the present use of them, as the observations are merely comparative. 
It would have been possible to utilize the actual oxygen consumption, expressed in cubic centimeters with as satisfactory results. The thyroxin used was Squibbs crystals of the highest grade. It was given in doses of $1 \mathrm{mg}$. after being put in solution in distilled water by rendering it slightly alkaline with dilute sodium hydroxide. The Lugol's solution (Liquor Iodi Composition) was given in doses of $0.1 \mathrm{cc}$. by mouth.

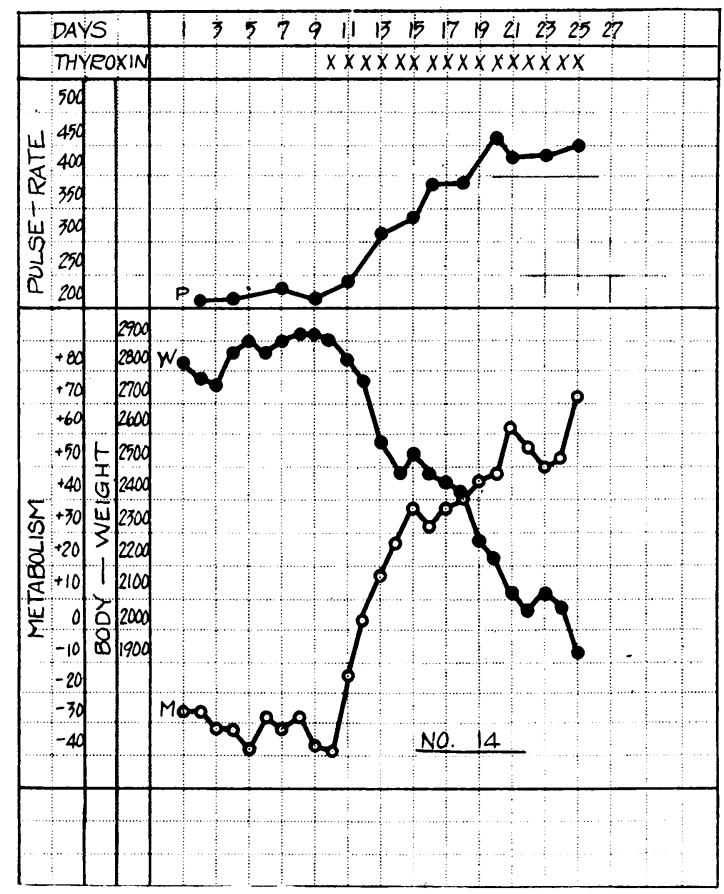

Chart 1. Experment 14. The Effect of 1 mg. of Thyroxin Intravenousiy for Sixteen Consecutive Days.

$P$, pulse-rate recorded in beats per minute; $W$, body-weight in grams; $M$, metabolism in per cent of normal; $X, 1 \mathrm{mg}$. of thyroxin intravenously.

THE EFFECT OF THE INTRAVENOUS INJECTION OF THYROXIN IN RABBITS

Chart 1 (experiment 14) illustrates the effect of the administration of thyroxin over a period of sixteen days. After a control period of nine days in which the metabolism and body-weight was recorded 
daily, and the pulse rate determined on four days of the period, the thyroxin injections were begun in doses of $1 \mathrm{mg}$. intravenously and continued for sixteen consecutive days as indicated by the cross marks at the chart. It will be noted that during the control period the pulse-rate remained very constant, the range being between 210 and 230 per minute. The body-weight was likewise fairly constant, although there was a slight tendency for the animal to gain weight.

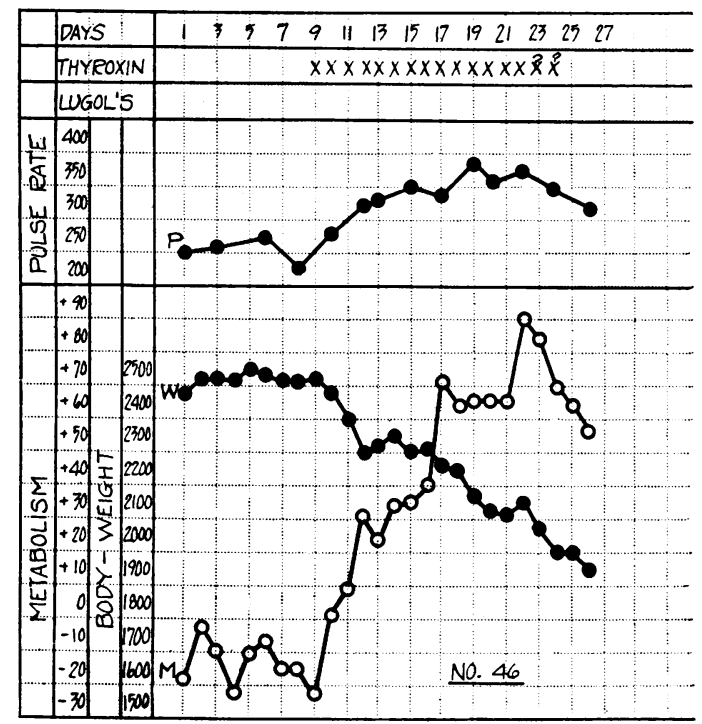

Chart 2. Experiment 46. The Effect of Repeated Milligram Doses of Thyroxin Intravenously on the Pulse-rate, Body-Weight and METABOLISM

As there was an immediate drop in weight following the use of the thyroxin, this made the effect even more convincing. During the control period the basal metabolism varied as much as 12 per cent, although the variations from one day to the next was very much less, and there was no constant tendency for the metabolism curve to proceed upward or downward. Following the injection of thyroxin there was an effect, slight but definite, within twenty-four hours as indicated by an accelerated pulse-rate, a decrease in bodyweight, and an increased metabolism. As the drug was continued there 
was a remarkable symmetrical rise in the pulse-rate which was paralleled by the metabolism. After the thyroxin had been injected for sixteen consecutive days, the pulse-rate was 450 per minute, and the metabolism had increased from -39 to +71 . Coincident with these changes the body-weight diminished from $2900 \mathrm{gm}$. to $1925 \mathrm{gm}$., which represents a loss of $975 \mathrm{gm}$. or approximately 35 per cent of the original body-weight. Although the animal's food consumption was only measured roughly, it was apparent that a normal amount

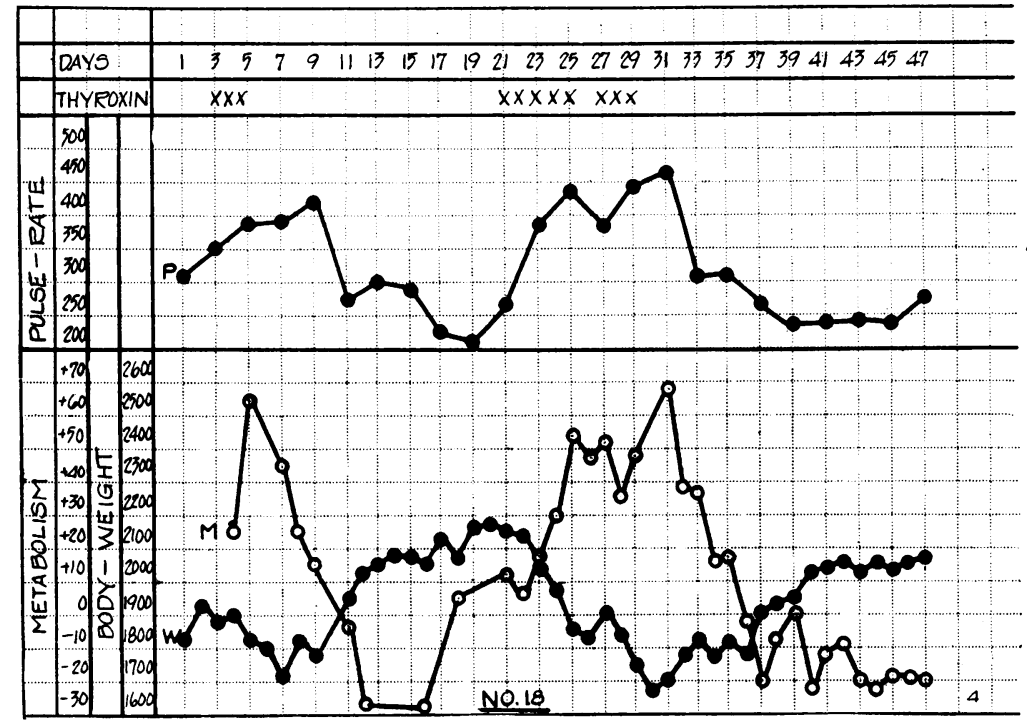

Chart 3. Experment 18. The Effect of Thyroxin Which Was Given in Milligram Doses for Three Consecutive Days, Then Omitted for Fifteen DAys, AND Resumed For Eight Doses

was consumed during the major portion of the experiment, although much less was eaten during the last few days of observation. It was obvious, however, that the chief cause of the loss of weight was due to the elevated metabolism. The experiment was terminated on the twenty-fifth day as it was clear that the characteristic effects of thyroxin had been demonstrated. These results were duplicated in experiment 46 (chart 2) in which $1 \mathrm{mg}$. of thyroxin was given for fourteen consecutive days after a satisfactory control period of eight 
days. The thyroxin was successfully given on twelve days. On the succeeding two days, attempts were made to administer the drug but probably only a small amount was actually injected as the ear veins were in poor condition. Chart 3 (experiment 18) shows the characteristic response to thyroxin, first, following its injection on three consecutive days, and again after an interval of sixteen days, at which time the drug was given in milligram doses for eight days. The striking increase in pulse-rate and basal metabolism when considered with the rather abrupt drop in body-weight, which follows shortly after the injection of thyroxin intravenously, appears to be entirely convincing that this drug is responsible for these changes and indicates that a rabbit will respond with characteristic alterations in pulse-rate, basal metabolism and body-weight after thyroxin is administered in doses similar to those used in the above experiments.

THE EFFECT OF THE SIMULTANEOUS ADMINISTRATION OF THYROXIN AND IODINE

Experiment 14 (chart 4) shows observations on an animal over a period of forty-nine days. This chart shows, first, the customary changes in the pulse-rate, basal metabolism and body-weight which are associated with the intravenous injections of thyroxin on three consecutive days. After this response had subsided, Lugol's solution, by mouth, was begun in daily doses of $0.1 \mathrm{cc}$., well diluted with water, and continued throughout the experiment. After this dosage had continued for eight days, thyroxin was also given daily in milligram doses for eight days; the animal received, therefore, $0.1 \mathrm{cc}$. of Lugol's solution and $1 \mathrm{mg}$. of thyroxin daily for this period. A comparison with the alterations in pulse-rate, basal metabolism and bodyweight in the two periods during .which the thyroxin was administered, shows that while the changes in the second period may be slightly greater than in the first, yet at this time more than twice as much thyroxin was given, and the animals response was in every way similar to that elicited in an animal by the injection of a similar amount of thyroxin and to which Lugol's solution had not been given (see chart 3). It is apparent from this chart that the animal gained considerably in body-weight during the forty-nine day period of study. This is most readily explained by the observation that con- 


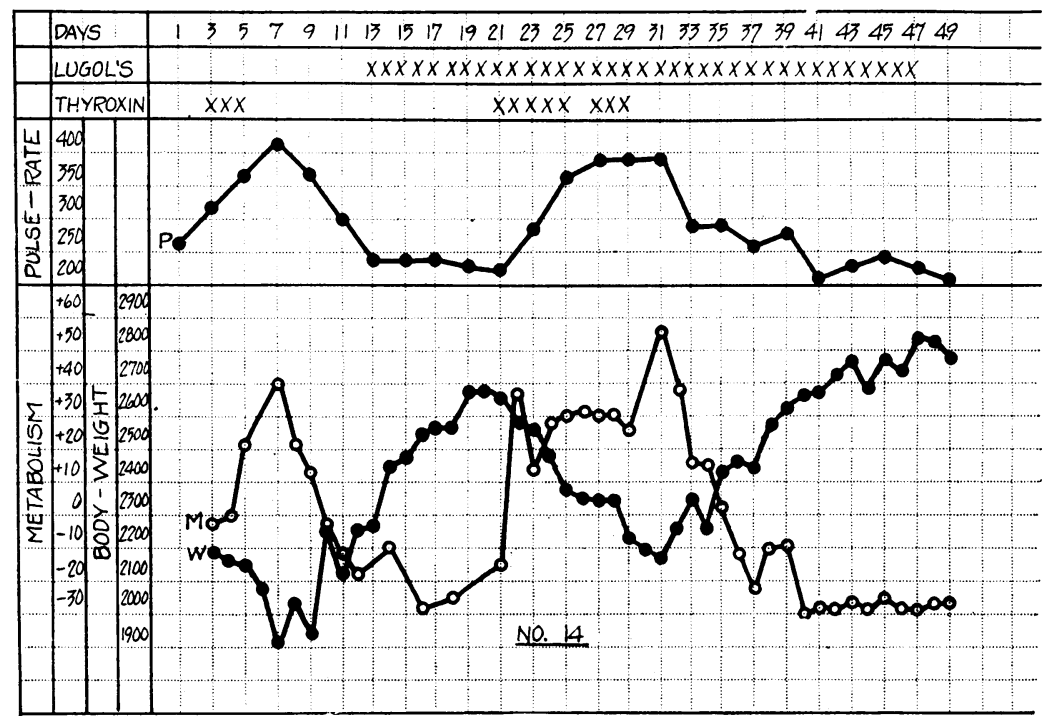

Chart 4. Experiment 14. Illustrating That Iodine by Mouth Does Not Effect the Response to Thyroxin

At the top of the chart, $X$ following Lugol's, indicates that $0.1 \mathrm{cc}$. was given by mouth on that day.

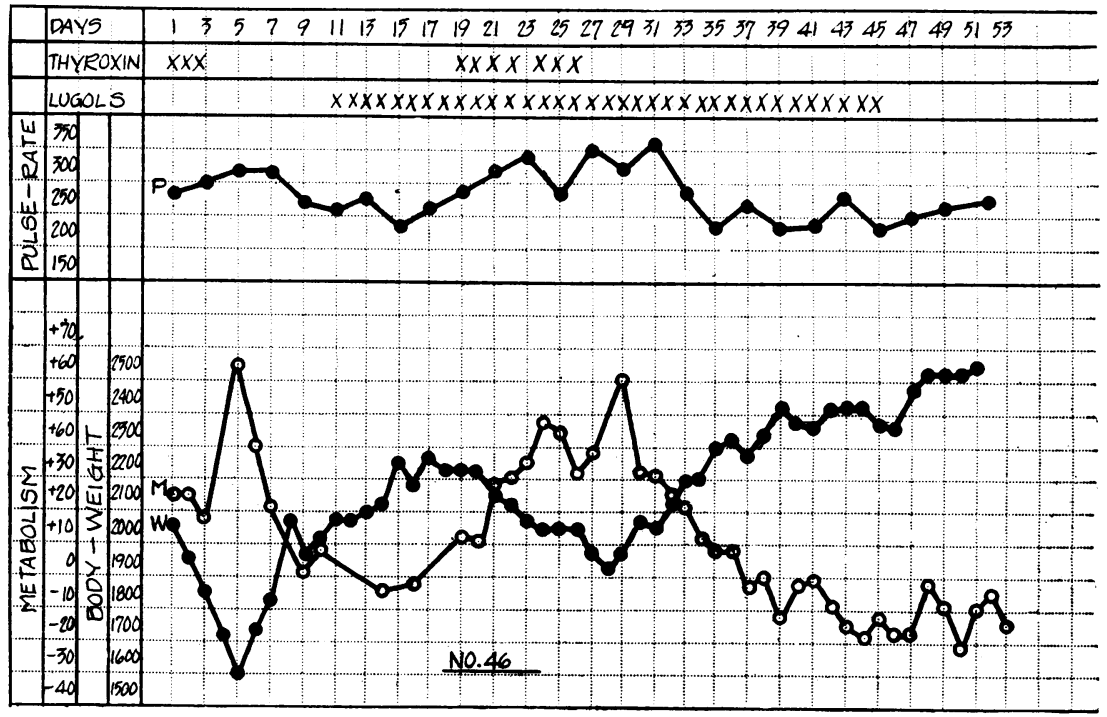

Chart 5. Experment 46. A Duplicate of the Results Shown in Chart 4 
trol animals in the laboratory gained a similar amount of weight which indicated that the environment, in which all of the animals were kept, permitted this. Experiment 46 (chart 5) is a duplicate of experiment 14 , and confirms in every respect the changes which had previously been observed. Additional studies were made which amply confirmed these experiments and indicated that observations in this direction would be a needless duplication of results.

\section{DISCUSSION}

From these experiments it is apparent that striking changes in the pulse-rate, basal metabolism and body-weight may be produced in rabbits by the intravenous injection of thyroxin. These changes are characteristic as they appear regularly following the injection of the drug, and they are similar to changes observed in patients with exophthalmic goiter although it can not be stated that the typical syndrome of the disease has been produced in animals. Additional observations indicate that the saturation of an animal with iodine by mouth, in the form of Lugol's solution, had no effect on the anticipated response to injections of thyroxin. If thyroxin is the active principle of the thyroid gland which is now generally accepted and it is assumed that the syndrome of exophthalmic goiter results from a pure excess of the substance, then it seems clear, from these experiments, that iodine has no effect on the circulating thyroxin, but must exert its influence on the rate of production of this substance in the gland itself. In addition, judging from a few unreported observations made in this laboratory, there is no evidence which suggests that iodine by mouth, at least in normal individuals, diminishes the normal rate of secretion of the thyroid gland, as the administration of iodine in man is not followed by a decrease in the basal metabolism. The experiments which are reported in this article are not incompatible with Plummer's theory (5) that the symptoms of exophthalmic goiter are due to an altered secretion of the thyroid gland and that this abnormal secretion may become more nearly normal following the administration of iodine. They furnish no support to it, however, except that further evidence is produced which is in accord with the theory that exophthalmic goiter is not due to a pure excess of thyroid secretion. 


\section{CONCLUSIONŚ}

1. The intravenous injection of thyroxin in rabbits produces a characteristic increase in the pulse-rate, basal metabolism and a decrease in the body-weight.

2. The administration of Lugol's solution has no effect in altering the characteristic response, in rabbits, to injections of thyroxin.

\section{BIBLIOGRAPHY}

1. Cattell, Richard B.: Boston Med. and Surg. Jour., 1925, cxcii, 989. The Pathology of Exophthalmic Goiter. A Histological and Chemical Study of the Changes Following the Administration of Iodine (Lugol's Solution).

2. Carpenter, Thorne M., and Fox, Edward L.: Boston Med. and Surg. Jour., 1923, clxxxix, 551. Alcohol Check Experiments with Portable Respiration Apparatus.

3. DuBois, Eugene F.: Basal Metabolism in Health and Disease. Philadelphia and New York, 1924, p. 39.

4. Krogh, August: The Respiratory Exchange of Animals and Man. London, 1916, p. 142.

5. Plummer, H. S.: Jour. Amer. Med. Assoc., 1923, lxxx, 1955. Results of Administering Iodine to Patients Having Exophthalmic Goiter. 